\title{
EDUCACIÓN, MERCADO Y CIUDADANÍA *
}

\author{
Juan Carlos Tedesco
}

\section{Introducción}

1. Vivimos un momento histórico particular, caracterizado por transformaciones sociales, culturales, económicas y políticas muy profundas, debates intensos y futuros no escritos. En definitiva, ningún país, ninguna sociedad, está satisfecha con la oferta educativa disponible y nadie está en condiciones de brindar respuestas categóricas a las preguntas que plantean las nuevas circunstancias sociales. Resulta crucial, por ello, reflexionar desde la duda, desde los interrogantes y no, como estamos acostumbrados, desde la pretensión de brindar una respuesta única y categórica a los problemas que enfrentan nuestras sociedades. No aceptar la duda, no aceptar que puede existir más de una solución al mismo problema, está provocando la expansión de fundamentalisrnos de diversos tipos, que dan lugar tanto a visiones que nos aseguran un destino maravilloso como a visiones que preanuncian catástrofes apocalípticas. Aceptar la duda, la incertidumbre y la necesidad de diversificar las soluciones, es un principio necesario no sólo a nivel individual sino institucional. Estimo que la sociedad del futuro, sometida a un ritmo acelerado y constante de cambio, debería dotarse de instituciones capaces de manejar la incertidumbre, sin apelar a la supresión del debate. La experimentación, admitida hasta hoy solamente como pauta de la investigación científica, debería tal vez comenzar a ser admitida en la práctica política.

2.Los conceptos de mercado, equidad y ciudadanía pueden ser objeto de múltiples interpretaciones filosóficas, sociológicas, políticas o económicas. Frente a la vastedad de esta problemática, he optado por referirme específicamente a algunos aspectos directamente vinculados a la dimensión educativa. Organizaré mi exposición alrededor de tres puntos centrales:

(i) El proceso de toma de decisiones educativas, donde intentaré discutir la opción entre el mercado y las modalidades de concertación y negociación como mecanismos de asignación de recursos y de cuotas de acceso a la educación.

(ii) El problema de la equidad en la distribución de la educación, donde trataré fundamentalmente del rol del Estado y del sector privado.

(iii) El problema de los contenidos de la educación, donde intentaré analizar el problema de la formación ciudadana y la formación para la competitividad económica.

Los tres temas están muy vinculados entre sí y en cada uno de ellos se hará referencia a los otros. Espero, sin embargo, que la claridad de la exposición no se vea afectada por este deseo de respetar el carácter complejo de los problemas que enfrentamos.

\section{Mercado y toma de decisiones}

3. Reconocer la necesidad de que las estrategias de acción educativa sean diseñadas a través de la participación de todos los actores sociales, ya es un lugar común en la literatura sobre políticas educativas. La continuidad en la aplicación de las estrategias de

\footnotetext{
"Este ensayo se basa en la presentación realizada en el panel "Educación, Estado Y Mercado", que tuvo luGar en el Foro escuela Siglo XXI (Bogotá, abril 3 de 1998).

* Director de la sede Regional del Instituto Internacional de Planeamiento de la Educación, Buenos Aires (Argentina).
} 
transformación ha sido reconocida como una de sus condiciones de éxito y para que exista continuidad, al menos en contextos democráticos, es necesaria la existencia de un nivel básico de acuerdo y de compromiso de todos los actores en su aplicación. En este sentido, la década del 90 se inició con cierto optimismo acerca de la existencia de condiciones favorables para la definición de estrategias educativas a través del consenso de los diferentes actores. Dicho optimismo se puso de manifiesto en la Conferencia Internacional "Educación para Todos", cuyos documentos postularon la necesidad y la posibilidad de establecer nuevas alianzas en favor de la educación ${ }^{200}$.

4. El factor clave de este optimismo fue la constatación según la cual, en los nuevos escenarios sociales, el conocimiento y la información constituyen las variables centrales tanto desde el punto de vista de la competitividad económica como desde la perspectiva del desempeño ciudadano y de la equidad social. Para el caso de América Latina, el documento elaborado a comienzos de esta década por la UNESCO y la CEPAL es un ejemplo del optimismo con el cual se concebía el rol de la educación y las posibilidades de definir acuerdos para la acción ${ }^{201}$. El punto de partida de este enfoque consistió en reconocer que la educación constituye una, y tal vez la única, variable de las políticas sociales que tiene la virtud de impactar simultáneamente sobre la competitividad económica, la equidad social y el desempeño ciudadano.

5. Pero a poco de iniciado este proceso se puso de manifiesto, sin embargo, que ir más allá del reconocimiento retórico acerca de la necesidad de concertar las políticas educativas, implicaba superar dificultades muy importantes. Si bien algunas de estas dificultades parecen propias de la escasa cultura política de concertación que existe en nuestros países, la hipótesis que desearía discutir con ustedes consiste en sostener que el origen de las dificultades radica en la propia centralidad que ocupa el conocimiento en la estructura social. Para decirlo en pocas palabras, creo que en la medida que la información y el conocimiento constituyen cada vez más las variables claves de la distribución del poder, el control de su producción y de su distribución se convierten en el ámbito donde se desarrollan, y se desarrollarán mucho más en el futuro, los conflictos sociales más significativos ${ }^{202}$.

6. Al respecto, la evolución social más reciente ha permitido apreciar que, contrariamente a los pronósticos de las hipótesis optimistas sobre las potencialidades democráticas de una economía y una sociedad basada en la producción de conocimientos $^{203}$, las economías productoras de ideas parecen ser más inequitativas que las que fabrican objetos. Tal como lo expresara recientemente A. Cohen, la propensión a excluir a los que no tienen ideas es más fuerte que la propensión a excluir a los que no tienen riquezas. La lógica que operaría en las economías productoras de conocimientos es la lógica de la calidad total o la del "error 0". En este tipo de funcionamiento, el menor error pone en crisis el conjunto de la cadena de producción, razón por la cual las calificaciones de los trabajadores en todos los puestos de trabajo deben ser muy altas. En esta lógica de funcionamiento, como sostiene Cohen, los mejores se juntan con los mejores y los mediocres con los mediocres. Las nuevas tecnologías exacerban esta

\footnotetext{
200 Meeting Basic Learning Needs: A Mission for the 1990 s. World Conference on Education for All. Background Document, Jomtien, Thailand, 5-9 march, 1990.

${ }^{201}$ Para el caso de América Latina, una presentación de este modelo de análisis puede verse en CEPAL UNESCO. Educación y conocimiento, bases de la transformación productiva con equidad. Santiago de Chile, 1991.

${ }^{202}$ Véase Juan Carlos Tedesco, el nuevo pacto educativo: competitividad y ciudadanía en la sociedad moderna, Madrid, Alauda-Anaya, 1995.

${ }^{203}$ Una versión muy popular de estas hipótesis optimistas puede verse en Alvin Toffler. El cambio del poder, Barcelona, Plaza y Janés, 1990.
} 
tendencia más o menos natural, al favorecer la descentralización, la externalización de actividades y el achatamiento de las pirámides de organización jerárquica de las unidades de producción ${ }^{204}$.

7. En este nuevo escenario, la segmentación se establece entre bloques completos de unidades productivas y no entre sectores al interior de una determinada empresa o sector de producción. La miseria del capitalismo contemporáneo consiste en crear, en el seno mismo de cada grupo social, tensiones que hasta ahora estaban en el ámbito de las rivalidades inter-grupos ${ }^{205}$. Esta dinámica del proceso productivo explica la aparente paradoja a la que confronta la observación de los procesos sociales contemporáneos, donde se advierte que el nuevo modo de producción se caracteriza por producir más igualdad y más desigualdad simultáneamente. Entre los que se incorporan al proceso productivo tecnológicamente más avanzado, existe mucha más homogeneidad que en el pasado, pero entre ellos y el resto de las personas que se desempeñan en unidades productivas tecnológicamente atrasadas o que son excluidas del proceso productivo, se establecen distancias mucho más significativas.

8. Las consecuencias de esta hipótesis según la cual la desigualdad asociada a un factor de la naturaleza humana la inteligencia pueden ser mucho más graves que la desigualdad asociada a un factor social —el poder, la riqueza, etc.-, son muy significativas y su discusión recién está comenzando. Mi deseo consiste, más modestamente, en advertir que en este nuevo contexto, la concertación sobre las políticas educativas, o sea, sobre las políticas relativas a la producción y la apropiación del capital más importante desde el punto de vista económico y social, no puede ser un proceso exento de dificultades y de conflictos duros. Al contrario, la experiencia reciente nos indica que la concertación es rechazada o resistida particularmente desde dos lugares diferentes. El primero de ellos es el que proviene de los enfoques económicos y políticos de inspiración "neo-liberal", según los cuales las decisiones educativas no pueden someterse a procesos de concertación ni de negociación, sino que deben ser dejadas como el resto de las decisiones sobre distribución de bienes y servicios a los mecanismos del mercado.

9. El segundo proviene de la discusión sobre los contenidos culturales que transmite la educación, donde las políticas educativas enfrentan la necesidad de concertar acerca de las posibilidades que tiene cada actor social para introducir determinados valores y concepciones del mundo en la educación, entendida como parte del proceso de socialización de las nuevas generaciones. En este ámbito asistimos también a fenómenos simultáneos de uniformidad y de aumento de la diversidad cultural. Los medios de comunicación de masas, particularmente a través de la enorme concentración de la producción de programas por parte de las empresas americanas, están promoviendo una homogeneización creciente en los estilos de vida de las personas. Pero al mismo tiempo, la uniformización cultural exacerba la necesidad de diferenciación, estimulando la multiplicación creciente de los factores de diversidad cultural.

IO. No es éste el lugar para un análisis del problema del multiculturalismo y la escuela. Simplemente, quiero destacar el hecho que el debate sobre los contenidos culturales que se transmiten a través de las instituciones educativas es mucho más complejo que en el pasado y que la concertación como metodología para resolver este problema es ya, en sí misma, una respuesta. Concertar políticas educativas desde el punto de vista curricular

\footnotetext{
${ }^{204}$ Albert Cohen, Richesse du monde, pauvreté des nations, Paris, 1997.

${ }^{205}$ A. Cohen, op. cit. 
constituye, en consecuencia, una forma específica de resolver los conflictos y las tensiones entre las diferentes opciones. En este ámbito, la concertación es atacada desde los enfoques fundamentalistas que reivindican la cohesión grupal sobre la base de algún criterio adscriptivo (religioso, étnico, etc.), y que consideran al diferente como un enemigo al cual es preciso eliminar. Este fundamentalismo de tipo colectivo no es la única fuente de rechazo a la concertación curricular. El rechazo también proviene de los fundamentalistas del individualismo a-social, que rechazan cualquier opción pública de formación cultural que establezca el contacto con el diferente, la solidaridad y la obligación de tener en cuenta al otro como un componente importante de la socialización escolar).

11. En síntesis, la diferencia fundamental entre la concertación de políticas educativas y las alternativas del mercado por un lado o del fundamentalismo autoritario por el otro, radica en el papel que se asigne a la dimensión política. Apelar a la concertación implica resguardar la esfera de la política en la toma de decisiones, ya que obliga a cada actor social a discutir y negociar públicamente sus opciones educativas. El mercado, en cambio, suprime la política y deja la toma de decisiones librada al resultado de decisiones individuales en función de intereses particulares y de corto plazo. El fundamentalismo autoritario, a su vez, elimina la política porque deja todo el poder en manos de un solo actor social. Obviamente, la concertación no es un proceso fácil. Existen numerosas cuestiones para resolver, entre las cuales se destacan dos: garantizar una representación adecuada especialmente de aquellos que no están organizados, y superar los consensos puramente retóricos para pasar a la adopción de acuerdos para la acción.

\section{Educación y equidad social}

12.Distribuir la educación en forma equitativa es una exigencia social, política, ética y económica. Asumo que la equidad como objetivo de la política educativa está fuera de discusión y' que el debate se ubica en las estrategias más eficaces para lograrlo. Al respecto, es oportuno recordar que las estrategias clásicas destinadas a promover la equidad social se basaban en lo que John Rawls llamó el "velo de la ignorancia"206. De acuerdo a este supuesto, la educación - como la justicia - debía organizarse sobre la base de ignorar las características particulares de cada persona. Tratar a todos de la misma manera, independientemente de sus condiciones particulares, fue el principio teórico básico de la acción educativa tradicional. Obviamente, en el funcionamiento real de las instituciones educativas, esta igualdad estuvo lejos de ser efectiva, pero las discriminaciones que aparecían en la realidad eran consideradas como ilegítimas, producto del mal funcionamiento del sistema y no como un producto natural de su dinamismo interno.

13. Es esta "opacidad de lo social", como condición de las políticas de equidad social lo que actualmente está desapareciendo. Por un lado, porque existe una reivindicación de las diferencias que resiste la idea de tratamiento homogéneo. Por el otro, porque existe un conocimiento mucho mayor de las diferencias que en el pasado y allí donde ese conocimiento no existe, la demanda por conocer es cada vez más intensa. Este fenómeno abre nuevas perspectivas a las políticas sociales pero también plantea nuevos riesgos y desafíos. Mantener el "velo de la ignorancia" provoca discriminaciones y exclusiones por todos conocidas. Tratar igual a los diferentes implica que aquellos que no se ajustan al modelo de tratamiento propuesto, fracasan y quedan fuera del acceso a los beneficios de las políticas de equidad. En educación, este fenómeno ha sido muy bien estudiado en los años 60 y 70, a través de los análisis sobre la expansión educativa y sus resultados. Pero

\footnotetext{
${ }^{206}$ John Rawls, Teoría de la Justicia, México, Fondo de Cultura Económica, 1979. 
este modelo también tuvo una importante función socializadora e integradora, apoyada en la existencia de un mecanismo común a todos. Levantar el velo de la ignorancia" tiene, en este sentido, ambas potencialidades. Permite comenzar a tratar a cada uno en función de su especificidad y de sus necesidades, pero también permite la des-solidarización basada en el conocimiento de las posibilidades y de los intereses de cada uno. Como advierte Pierre Rosanvallon, el conocimiento de la especificidad y su re-conocimiento genera un nuevo principio de vida social donde la tolerancia reemplaza la solidaridad y la imparcialidad reemplaza la igualdad y la equidad.

14. La mayor transparencia en las políticas sociales genera, por lo tanto, mayores niveles de complejidad, de flexibilidad y de inestabilidad en las estrategias de acción. Así, por ejemplo, el diseño institucional de la acción educativa debe enfrentar el dilema de otorgar mayor autonomía a los establecimientos para adecuarse a la diferenciación social y cultural existente y, al mismo tiempo, evitar que la adecuación a las diferencias se convierta en adecuación a la desigualdad y se rompa la cohesión social mínima indispensable para la existencia de una vida en común. Estas alternativas, estas opciones diferentes, estos dilemas, son la fuente de numerosos malos entendidos, confusiones y ambigüedades que caracterizan los actuales debates educativos. Respeto a la diversidad y autonomía en la gestión han sido frecuentemente asociados o concebidos como sinónimos de privatización o de desresponsabilización del Estado. Políticas de equidad y de compensación han sido calificadas como resabios del pasado, del populismo demagógico o del estatismo ineficiente. Es preciso, en consecuencia, realizar un significativo esfuerzo de clarificación conceptual y política que permita superar la confusión y que oriente el comportamiento de los distintos actores sociales frente a las opciones de políticas educativas que se abren para el futuro.

15. En primer lugar, me parece importante distinguir dos conceptos: el concepto de diversidad y el concepto de desigualdad. En América Latina estas dos categorías han estado - y lo están aún muy asociadas. Los diferentes son desiguales. Pero esta asociación empírica no justifica que tratemos las dos situaciones como similares. El objetivo de toda política educativa y social democrática es atacar la desigualdad, no la diversidad. La confusión de ambos conceptos lleva a menudo a pensar estrategias donde el respeto a la diversidad y la personalización de los enfoques sólo se conciben como válidos para las acciones dirigidas a las capas medias y altas de la sociedad, mientras que para los sectores populares seguimos actuando con estrategias masivas, suponiendo que en estos sectores no existe diversidad ni pluralismo. Este enfoque condena las políticas públicas a moverse en el camino de la uniformización, de la despersonalización y de la simplificación. La complejidad también existe en situaciones de pobreza, donde lo único pobre es la disponibilidad de recursos materiales.

16. En segundo lugar, es preciso aclarar cierta confusión en cuanto a la relación entre educación y equidad social. Se ha discutido y se sigue discutiendo con intensidad si la educación es la variable fundamental para explicar y para superar la pobreza. Muchos piensan que en lugar de otorgar tanta importancia a la educación, sería mejor modificar la distribución del ingreso, las condiciones del comercio internacional o la corrupción de la clase política. El debate, sin embargo, no pasa por decidir si una variable es más o menos importante que otra. Las estrategias de desarrollo social - se ha dicho repetidamente tienen que ser estrategias sistémicas. Pero para el caso que nos ocupa es importante advertir que la relación de determinación entre educación y equidad social es recíproca. La educación contribuye sin duda a la equidad social. Sin educación es imposible acceder a puestos de trabajo productivos y es imposible participar activamente en la vida ciudadana. Pero sin equidad social es imposible educarse adecuadamente. Todos los 
análisis sobre rendimiento escolar muestran que para educarse y para aprender, son necesarios niveles básicos de nutrición, salud y condiciones materiales de vida. Así como hoy en día se habla cada vez más de empleabilidad, para referirse a las condiciones que una persona debe tener para poder competir en el mercado de trabajo, también es posible hablar de educabilidad, para referirnos a las condiciones mínimas que una persona debe tener para ser educada. Las políticas de equidad social son, en este sentido, una condición necesaria de las políticas educativas. En este sentido, es legítimo reclamar a los que demandan más eficiencia en la gestión educativa, que se ocupen también de garantizar las condiciones básicas de educabilidad para que esa eficiencia sea posible.

17. En tercer lugar, estimo importante ubicar este debate sobre educación y equidad social en el marco de las actuales transformaciones económicas, sociales y culturales. Desde este punto de vista, el papel de la educación en la equidad social está asumiendo características más relevantes que en el pasado, debido al cambio en el papel del conocimiento y la información en la determinación de la estructura social. En este sentido, es importante retomar los planteos de André Gorz $^{207}$ frente a la evolución del trabajo en la sociedad. La mejor y tal vez la única estrategia para enfrentar democráticamente la tendencia a la exclusión que muestra la sociedad consiste en que todos estén educados para desempeñarse en el sector moderno del mercado de trabajo. De otra manera. estaríamos frente a un escenario de neodespotismo ilustrado, donde unos pocos tendrían los conocimientos capaces de regular el funcionamiento de la sociedad, frente a una masa de excluidos sin competencias para competir por el control de las herramientas de poder.

18. Las estrategias educativas destinadas a garantizar niveles adecuados de equidad han estado asociadas estrechamente al rol de Estado. Actualmente, sin embargo, es frecuente escuchar propuestas en favor de diversas formas de privatización de la educación como alternativa más eficaz, más barata, de utilización de los recursos públicos en educación. El análisis de la información empírica disponible pone en evidencia que buena parte de los argumentos que se utilizan en estas discusiones siguen siendo argumentos de fuerte carácter ideológico .En este sentido, repito, el análisis de la información empírica permite demostrar al menos tres hipótesis muy importantes. La primera de ellas es que no existe una asociación unívoca entre privatización de la educación, modernización y desarrollo social. La segunda es que no existe una asociación unívoca entre privatización y desregulación del funcionamiento de las instituciones educativas, y la tercera es que no existe asociación unívoca entre privatización y mejores resultados de aprendizaje.

19. Con respecto a la asociación entre público, privado y desarrollo social, los datos sobre evolución de la enseñanza pública y privada en las últimas décadas permite apreciar que existe una gran diversidad de situaciones pero que, en términos generales, la mayor importancia de la educación privada está asociada a menores niveles de desarrollo social y no a la inversa. No es el caso aquí de hacer un detallado análisis estadístico, pero quisiera simplemente ilustrar estas afirmaciones con el caso de la enseñanza secundaria. Mientras en los países desarrollados el sector privado atiende un porcentaje cercano al $15 \%$ de la matrícula (semejante al registrado en la enseñanza primaria), el porcentaje de matrícula privada en los países en desarrollo alcanza casi el 30\%.

20. En cuanto a la relación entre escuela privada y control público, también el análisis de la situación internacional permite apreciar que los casos más exitosos de desarrollo de

${ }^{207}$ A. Gorz, Métamorphoses du travail; Quéte du sens. Critique de la raison économique, Paris. Galilée, 1988. 
la educación privada están asociados a fuertes regulaciones públicas. En este sentido, me parece interesante recordar el caso de la experiencia holandesa, país donde las fórmulas de combinación entre el sector público y el sector privado han demostrado ser muy eficaces. Históricamente. el origen de la demanda por enseñanza privada estuvo fuertemente vinculado a la heterogeneidad religiosa. Tanto la comunidad católica como la protestante demandaron al Estado el apoyo para poder brindar a sus hijos una educación en el marco de sus códigos culturales. "Separados pero iguales» fue la fórmula que resumió esta demanda de respeto a la diversidad por un lado y de equidad social por el otro.

21. Para garantizar este objetivo, las fuertes regulaciones del Estado fueron fundamentales. Desde el punto de vista del financiamiento, por ejemplo, el Estado brinda un subsidio pero limita severamente la posibilidad de establecer otros pagos adicionales; paga todos los salarios de los docentes, tanto del sector público como del sector privado, pero no permite a las escuelas dar suplementos saláriales; provee los edificios, tanto para escuelas públicas como privadas, a través de los municipios, pero con reembolso del gobierno central. Las cuotas cobradas por las escuelas privadas son muy bajas, debido tanto al temor a perder el subsidio estatal como a la fuerte competencia debido a la facilidad de entrada al mercado permitida por la ley. Por último, el gobierno mantiene fuertes regulaciones a través de insumos claves de la acción educativa: curriculum uniforme, sistema nacional de exámenes al final de la escuela elemental y de la escuela secundaria y controles en los criterios para seleccionar estudiantes ${ }^{208}$. El resultado de esta articulación es que las escuelas privadas holandesas no reclutan alumnos de orígenes sociales distintos a los de las escuelas públicas.

22. Por último, quisiera referirme al argumento según el cual la privatización es garantía de mejores resultados de aprendizaje, debido a la posibilidad que tienen las escuelas privadas de asumir mayores niveles de responsabilidad por los resultados y mayor dinamismo pedagógico. Los mejores resultados de las escuelas privadas deben, sin embargo, ser analizados teniendo en cuenta el origen social de los alumnos. La información disponible indica que, en general, la enseñanza privada recluta su alumnado en sectores medios y altos, propiciando de esta forma un fenómeno circular: alumnos dotados de mejores antecedentes familiares reciben una oferta escolar caracterizada por la disponibilidad de equipamiento y de personal adecuado, obtienen resultados más altos que los producidos por la escuela pública. Pero también existen casos donde la oferta privada se ha ampliado a sectores populares, sobre la base de subsidios estatales. El argumento para estimular este tipo de alternativas ha sido, habitualmente, un argumento financiero. Según algunos estudios, resultaría más barato para el Estado financiar un establecimiento privado que ofrezca educación gratuita, que financiar una escuela pública. De lo que se trata, ahora, es saber si además de la supuesta ventaja financiera, también existen ventajas desde el punto de vista de los resultados de la acción pedagógica.

23. Los resultados aportados por la información empírica disponible de algunos países de América Latina acerca de la acción educativa pública y privada indican que, en definitiva, la explicación de buenos resultados de aprendizaje no radica en el carácter estatal o privado de los establecimientos escolares sino en su dinámica institucional. Los mejores rendimientos están asociados a la posibilidad de definir un proyecto educativo del establecimiento escolar, definido por la conciencia de determinados objetivos, por la existencia de ciertas tradiciones y metodologías de trabajo compartidas, espíritu de equipo y

${ }^{208}$ Estelle James. «Benefits and Costs of Privatized Public Services: Lessons from the Dutch Educational Svstem». in Comparative Education Review, vol. 28, No. 4, 1984. 
responsabilidad por los resultados, es decir, por la identidad institucional. La dicotomía entre eficiencia y dinamismo como patrimonio del sector privado y rigidez e ineficiencia como patrimonio del sector público ya no son sostenibles. En realidad, no existe ninguna razón por la cual el sector público no pueda adoptar estrategias de acción que brinden a sus servicios, específicamente a su servicio educativo, características de dinamismo y responsabilidad por los resultados sin perder por ello su carácter socialmente democrático.

\section{Mercado, competitividad y ciudadanía}

24.Por último quisiera referirme al problema de los contenidos de la educación. No lo haré desde una perspectiva estrictamente curricular, muy importante por cierto, pero que supone que ya sabemos qué es lo que la educación formal debe transmitir. Lo haré, en cambio, desde la perspectiva del proceso de socialización de las nuevas generaciones, proceso en el cual la educación formal es uno de sus componentes, pero no el único. Desde este punto de vista, el fenómeno más importante que se percibe en la sociedad contemporánea, es la erosión de la capacidad socializadora del conjunto de las instituciones clásicamente responsables de esta función. Existe, exagerando un poco los términos, un problema de "déficit de socialización», de ausencia de experiencias que garanticen la incorporación a la sociedad con marcos de referencia relativamente consolidados $^{209}$. Las instituciones educativas tradicionales, particularmente la familia y la escuela, están perdiendo capacidad para transmitir los valores y las pautas culturales que aseguren los niveles básicos de cohesión de una sociedad. Con respecto a la escuela, es bien sabido que la cultura escolar se ha aislado significativamente de la cultura social y que frente al dinamismo del cambio social, la escuela ha permanecido relativamente estática e inmodificable. En cuanto a la familia, fenómenos tales como la incorporación de la mujer al mercado de trabajo, la tendencia a reducir el número de hijos, el aumento de separaciones, hijos que viven solos o sólo con uno de sus padres, han modificado su papel tradicional y han provocado una diversificación muy significativa de las formas institucionales que asumen las relaciones familiares. Si bien no es posible generalizar a todas las culturas la existencia de estos fenómenos, puede resultar interesante mostrar un caso extremo: en los EEUU, según datos recientes, si las tendencias actuales se mantienen, menos de la mitad de los niños y niñas nacidos hoy vivirán con su propia madre y padre durante su niñez y un número creciente de niños y niñas vivirán la experiencia de ruptura familiar dos o aun tres veces durante ese período. En las sociedades menos desarrolladas también es significativo el proceso de pérdida de poder socializador por parte de la familia, las familias pobres suelen ser familias donde la figura paterna está ausente y donde los niños pasan desde edades muy tempranas, períodos prolongados de tiempo sin la presencia de sus padres.

25. Este "déficit de socialización" producido por los cambios en la escuela y en la familia no ha sido cubierto por los nuevos agentes de socialización. Entre los nuevos agentes de formación cultural se destacan, obviamente, los medios masivos de comunicación y, en especial. la televisión. Sin embargo, los medios de comunicación no han sido diseñados como agencias encargadas de la formación moral y cultural de las personas. Al contrario, su diseño y su evolución suponen que dicha formación ya está adquirida y, por eso, la tendencia actual de los medios consiste en depositar en los ciudadanos mismos, la elección de los mensajes que quieren recibir.

\footnotetext{
${ }^{209}$ Las hipótesis presentadas en esta sección del artículo están desarrolladas más extensamente en Juan Carlos Tedesco, El Nuevo Pacto Educativo, Madrid, Anaya. 1995. 
26. La crisis de las instituciones socializadoras está íntimamente asociada a un fenómeno que los analistas de la sociedad contemporánea han llamado la "crisis de sentido". Siguiendo estos análisis es posible sostener que la sociedad actual habría perdido el sentido en varias dimensiones diferentes. En primer lugar, en términos del fundamento de la sociedad. Al respecto. el fin de la guerra fría habría marcado no sólo el fin del comunismo sino el fin de dos siglos de lluminismo, es decir, de la vigencia de un esquema conceptual, ideológico, político, que otorgaba sentido a la acción de todos los actores. La quiebra de este esquema conceptual se refleja en la dificultad para objetivar, para representar de alguna manera el futuro que plantea la mundialización y que permitiría invertir en ella por motivos afectivos, de adhesión a principios que vayan más allá de la mera necesidad económica. En segundo lugar, la pérdida de finalidades hace desaparecer la promesa social o política de un «futuro mejor». Como lo muestran numerosos análisis de la situación económica actual, la incapacidad del Estado para proteger a los ciudadanos y ofrecer una imagen de futuro no produce una transferencia de esta función a los ciudadanos mismos. Al contrario, esta ausencia estimula conductas de corto plazo que tienen su máxima expresión en el comportamiento del mercado financiero y en las presiones de los accionistas sobre el comportamiento de las empresas.

27. Las empresas se encuentran actualmente guiadas por los imperativos de conquistar mercados y reducir el tiempo entre innovación y comercialización de un producto. Pero esta doble dinámica se refiere más bien a los «senderos» de la competitividad y no a sus finalidades. El cambio tecnológico acelerado aparece no sólo como algo suscitado por la actividad económica sino también sufrido por ella. Como lo sostiene lúcidamente Z. Laídi, "todos los actores del juego social mundial se proyectan en el futuro no para defender un proyecto sino para evitar ser excluidos de un juego que no tiene rostro... El fin de la utopía ha provocado la sacralización de la urgencia, erigida en categoría central de la política. Así, nuestras sociedades pretenden que la urgencia de los problemas les impide reflexionar sobre un proyecto, mientras que en realidad es la ausencia total de perspectiva lo que nos hace esclavos de la urgencia"210.

28. La pérdida de sentido tiene consecuencias muy importantes sobre la educación, entendida desde el punto de vista del proceso de socialización, ya que deja a los educadores sin puntos de referencia. En las condiciones actuales, esta pérdida de sentido tiene, al menos, tres consecuencias importantes:

(i) En primer lugar, reduce el futuro y las perspectivas de trayectoria tanto individual como social a un solo criterio dominante, el criterio económico. Pero el criterio económico actual no tiene ni siquiera la capacidad inclusiva del capitalismo industrial. El modelo de desarrollo económico actual, sin el control y la regulación de las instancias políticas, provoca fenómenos crecientes de exclusión. Se quiebra, de esta manera, la posibilidad de la cohesión social, de transmitir un mensaje socializador donde cada uno encuentre su lugar. Este empobrecimiento del proyecto futuro provoca una baja capacidad de adhesión, erosiona todos los vínculos sociales y se convierte, en última instancia, en un proyecto asocial, un proyecto que elimina la centralidad de los vínculos políticos y de las lealtades ciudadanas en la formación de las personas.

(ii) Coloca la transmisión de las identidades, tanto culturales como profesionales o políticas en términos regresivos. Las dificultades para transmitir el patrimonio cultural del pasado en función de una línea de continuidad histórica con proyección de futuro, provoca la tentación del retorno a las visiones fijas y rígidas de las identidades del pasado.

${ }^{210}$ Op. cit., pág. 29. 
(iii) Como consecuencia de lo anterior, se fortalece el inmovilismo y se produce una fuerte desconfianza frente a toda idea de transformación. El imperativo de la transformación es vivido como contrario al imperativo de la transmisión de la identidad. La transmisión es juzgada como conservadora y la transformación es juzgada como destructora.

29. La educación vive esta situación de una manera particularmente dramática. No es ésta, por supuesto, la primera gran transformación de la sociedad ni, en consecuencia, la primera vez que el proceso de socialización de las nuevas generaciones implica un profundo proceso de reconversión social. Sin embargo, lo peculiar de este momento histórico es que las fuentes tradicionales de identidad han desaparecido y que las nuevas fuentes se caracterizan, precisamente, por la ausencia de puntos fijos de referencia. La identidad, por lo tanto, debe ser construida. Este es, probablemente, el concepto más importante para referirnos al proceso pedagógico que requieren los cambios sociales actuales. Los contenidos de la educación, en consecuencia, ya no pueden ser concebidos como paquetes elaborados disponibles para ser transmitidos, sino como capacidades que le permitan a cada uno construir su conocimiento. Aprender a aprender, en la fórmula utilizada recientemente por el Informe de la Comisión Delors, resume este objetivo educativo fundamental para el futuro.

30. La inclusión de estos temas en la agenda de discusión de políticas públicas sería, de por sí, un indicador de la madurez de los sectores dirigentes de una sociedad, en la medida que pone de relieve la voluntad de trascender el corto plazo y preocuparse responsablemente del legado que se deja a las futuras generaciones. En pocas palabras, lo que quisiera decir es que las estrategias educativas en este ámbito deberían partir del supuesto según el cual para superar el deficit de socialización que mencionamos antes, es preciso desarrollar políticas tendientes a incrementar significativamente las experiencias de aprendizaje ligadas al desarrollo de valores y actitudes ciudadanas. Lo interesante de este momento histórico es que las capacidades que requiere el desempeño ciudadano y las capacidades que requiere el desempeño productivo han superado la fase de contradicción que se vivía en el marco del capitalismo tradicional. Actualmente la solidaridad, el trabajo en equipo, la creatividad, el manejo de los códigos expresivos, etc., son capacidades indispensables para el desempeño social en general. En esta articulación podemos colocar buena parte del optimismo con respecto al futuro.

31. El debate está abierto. Nadie tiene respuestas categóricas pero, como ya lo dijeron hace algunos años los propios responsables de las políticas educativas latinoamericanas, es necesario reconocer que si bien estas visiones son visiones de largo plazo, ocuparse del largo plazo también es urgente. 